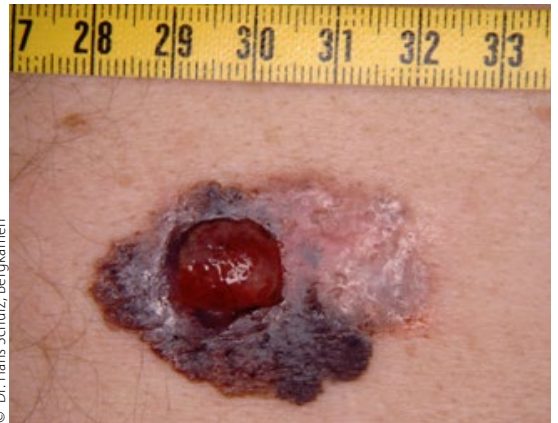

\title{
Kinasehemmer-Kombi als neuer Standard
}

Bei malignen BRAF-mutierten Melanomen wird sich wohl die Kombination aus einem BRAF-Inhibitor und einem MEKInhibitor als Standardtherapie etablieren. Zumindest sprechen die finalen Ergebnisse der COMBI d-Studie dafür, die im Vergleich zur ersten Analyse auf einem zusätzlichen 17-monatigen Follow-up (bis 34 Monate) basieren.

211 Patienten mit einem nicht resezierbaren oder metastasierten Melanom mit BRAF-V600K- oder BRAF-V600E-Mutation erhielten eine Kombinationstherapie mit dem BRAF-Inhibitor Dabrafenib (150 mg 2-mal täglich) und dem MEK-Inhibitor Trametinib (2 mg 1-mal täglich) im Mittel über 20 Monate, 212 Teilnehmer nahmen Dabrafenib mit Placebo ein (im Mittel 16 Monate).

Letztlich verlängerte die Kombinationstherapie das progressionsfreie Überleben im Vergleich zur Monotherapie sogar deutlicher, als es in der ersten Analy- se mit 9,3 Monaten zu sehen war: nämlich um 11 Monate im Vergleich zu 8,8 Monaten unter dem BRAF-Inhibitor. Das Sterberisiko für Patienten, die beide Kinasehemmer eingenommen hatten, reduzierte sich signifikant um 29\%, mit einem mittleren Gesamtüberleben von 25,1 Monaten verglichen mit 18,7 Monaten mit Dabrafenib allein. Die Überlebensrate betrug nach einem Jahr 74 versus $68 \%$ und nach zwei Jahren 51 versus $42 \%$. Auch die Ansprechraten waren unter der Kombination höher als unter der Monotherapie (69 vs. $53 \%$ ).

Die Nebenwirkungsrate unterschied sich in den beiden Behandlungsgruppen kaum. Es kam zu keinen unerwarteten Ereignissen. Fieber war die häufigste Nebenwirkung, die in Verbindung mit beiden Kinasehemmern auftrat (bei $52 \%$ ). Toxische Effekte, die auf die paradoxe Aktivierung des MAPK-Signalwegs zurückzuführen sind, wie Hyperkeratosen
Beim malignen BRAF-mutierten Melanom hat sich die Kombination zweier Kinasehemmer als überlegen erwiesen.

oder Zweittumoren (z. B. Plattenepithelkarzinome der Haut), sind mit der Kombination der beiden Substanzen seltener aufgetreten als mit der alleinigen BRAFInhibition.

Derzeit laufen weitere Studien, die die Wirkung einer Dreifachkombination aus Dabrafenib und Trametinib mit einer Immuntherapie untersuchen.

Veronika Schlimpert

Long GV et al. Dabrafenib and trametinib versus dabrafenib and placebo for Val600 BRAF-mutant melanoma: a multicentre, double-blind, phase 3 randomised controlled trial. The Lancet 2015;386 (9992):444-51.

\section{kurz notiert}

Forschungsstandort Deutschland

Antitumoraktivität von Bakterien +++ Einige fakultativ anaerobe Bakterien wie Escherichia coli können das Tumorwachstum bremsen oder den Tumor sogar zum Verschwinden bringen. Eine Arbeitsgruppe am HelmholtzZentrum für Infektionsforschung in Braunschweig fand nun heraus, dass $\mathrm{CD}_{4}^{+-}$und CD8+-T-Zellen für die Abwehr des Tumors sorgen. Auch die Expressionen von Granzyme B, FasL, TNF-a und IFN- $\gamma$ sind an der Antitumoraktivität von Bakterien beteiligt [Stern C et al. Int J Cancer. 2015;137(8):2019-28].

Hirnkartierung verbessert Prognose +++ Mit der navigierten transkraniellen Magnetstimulation (nTMS) lässt sich feststellen, welche Bereiche des Gehirns Bewegung oder Sprache steuern. Mediziner der Technischen Universität München konnten kürzlich nachweisen, dass eine nTMS-Analyse für Bewegungsareale vor der Operation die Prognose von Patienten mit hochmalignen Gliomen verbessert. Bei den Patienten mit nTMS-Analyse waren weniger große Schädeleröffnungen notwendig und nach dem Eingriff blieben seltener Tumorreste zurück. Zudem lebten diese Patienten länger als die Kontrollgruppe [Krieg SM et al. BMC Cancer. 2015;15:231]

\section{Ältere Patienten mit akuter myeloischer Leukämie MRD-negativ? Prognose dennoch schlecht}

Ein negativer Test auf eine minimale Resterkrankung (MRD) bedeutet für ältere Patienten mit akuter myeloischer Leukämie (AML) eine schlechtere Prognose als bei jüngeren. Das zeigte eine retrospektive Studie mit 210 AML-Patienten, von denen 149 jünger als 60 Jahre waren. Sie hatten nach der Induktionstherapie eine morphologische komplette Remission erreicht. Nach der Konsolidierungstherapie hatten signifikant weniger Ältere einen negativen MRD-Status (11 vs. $28 \%$; $p=0,009$ ).

In beiden Altersgruppen war die MRDNegativität mit signifikant höheren progressionsfreien Überlebensraten nach fünf Jahren assoziiert ( $<60$ Jahre: 56 vs. $31 \%$; $\geq 60$ Jahre: 57 vs. $13 \%$ ). Der Vorteil spiegelte sich auch in der Gesamtüberlebensrate wider $(<60$ Jahre: 57 vs. $31 \%$; $\geq 60$ Jahre: 71 vs. $16 \%$ ).

In beiden Gruppen war schließlich bei Patienten mit einem positiven MRD-Test die kumulative Inzidenz an Rückfällen
(CIR) jeweils in etwa verdoppelt $(<60$ Jahre: 59 vs. $24 \%$; $\geq 60$ Jahre: 83 vs. $42 \%$ ). Allerdings gab es bei der CIR einen wesentlichen Unterschied zwischen Alt und Jung, wenn der MRD-Test negativ ausfiel: In diesem Fall war die CIR bei den älteren Studienteilnehmern nach fünf Jahren fast doppelt so hoch wie bei den jüngeren (42 vs. $24 \%$ ).

Die Autoren gehen davon aus, dass dieser Unterschied biologische Gründe hat und auf dem Vorhandensein leukämischer Stammzellen beruht. Die höhere CIR hat auch Konsequenzen für die Behandlung. Eine Option könnte dann sein, die Therapie fortzusetzen und - altersgerecht - intensiver zu gestalten, um das Gesamtüberleben zu verlängern.PeterLeiner

Buccisano F et al. Minimal residual disease negativity in elderly patients with acute myeloid leukemia may indicate different postremission strategies than in younger patients. Ann Hematol. 2015; 94(8):1319-26. 